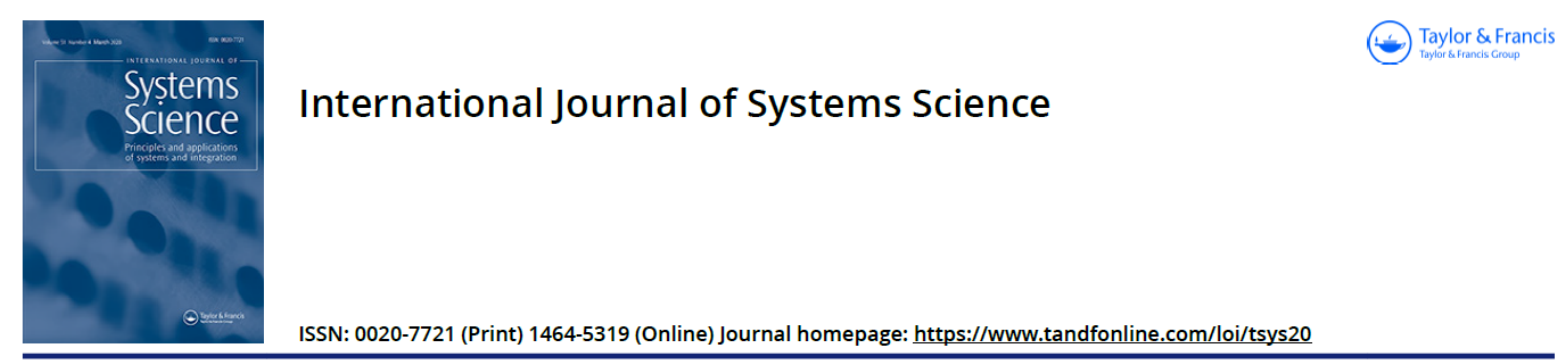

\title{
Least-squares estimators for systems with stochastic sensor gain degradation, correlated measurement noises and delays in transmission modelled by Markov chains
}

M. J. García-Ligero, A. Hermoso-Carazo \& J. Linares-Pérez

\section{Preprint version. Please cite original version:}

M. J. García-Ligero, A. Hermoso-Carazo, J. Linares-Pérez (2020). Least-squares estimators for systems with stochastic sensor gain degradation, correlated measurement noises and delays in transmission modelled by Markov chains, International Journal of Systems Science, 51 (4), 731-745. https://doi.org/10.1080/00207721.2020.1737757

\begin{abstract}
This paper addresses the linear least-squares estimation of a signal from measurements subject to stochastic sensor gain degradation and random delays during the transmission. These uncertainty phenomena, common in network systems, have traditionally been described by independent Bernoulli random variables.Wepropose a model that is more general and therefore has greater applicability to real-life situations. The model has two particular characteristics: firstly, the sensor gain degradation is represented by a white sequence of random variables with values in $[0,1]$; in addition, the absence or presence of delays in the transmission is described by a homogeneous three-state Markov chain, which reflects a possible correlation of delays at different sampling times. Furthermore, assuming that the measurement noise is one-step correlated, we obtain recursive prediction, filtering and fixed-point smoothing algorithms using the first and second-order moments of the signal and the processes present in the observation model. Simulation results for a scalar signal are provided to illustrate the feasibility of the proposed algorithms, using the estimation error variances as a measure of the quality of the estimators.
\end{abstract}




\section{Introduction}

The estimation of signals perturbed by additive noise has been widely studied, and various estimation algorithms have been proposed, most of which assume that the measurements always contain the true signal. Unfortunately, this assumption is unfounded in many modern systems, in which the signal measurements may be subject to random uncertainties due to physical constraints, measurement costs or environmental complexities. For example, aging or intermittent performance of the sensors can produce fading measurements, or even missing measurements, consisting only of noise. In addition, during data transmission via communication channels, faults may occur due to limited communication bandwidth, congestion or defects in the channels. These failures can produce random uncertainties in the processed measurements such as packet losses, caused by communication failures, or delays, caused by limited speed of information processing as occurs, for example, in chemical process. In these situations, conventional algorithms are not applicable and it is necessary to develop new ones that take these uncertainties into account. For example, estimation algorithms have been derived in Sun, Xie, Xiao \& Soh (2008), García-Ligero, Hermoso-Carazo \& Linares-Pérez (2011) and Guo (2017), for systems with packet dropouts; in Sun \& Xiao (2013), Caballero-Águila, Hermoso-Carazo, Linares-Pérez \& Nakamori (2013) and Sun \& Tian (2011), for random delays, and in Gao \& Chen (2014), Hu, Wang, Gao \& Stergioulas (2012) and Pang \& Sun (2015), considering missing measurements.

With respect to signal measurement failures, a specific problem that has received considerable attention is that of missing measurements. One approach to modelling the presence or absence of the signal in the measurements is to make use of Bernoulli random variables (see e.g. Caballero-Águila Hermoso-Carazo, Linares-Pérez, 2017a, and references therein). However, in many practical situations, faults in the sensor outputs may produce deteriorated signal measures, which cannot be simply described by random variables taking the values 0 (signal missing) or 1 (signal present in the measured output). A proposal that is generally accepted among the scientific community to describe this gain degradation phenomenon is to use random variables distributed over the interval $[0,1]$, which includes the possibility of missing measurements as a special case. In recent years, there has been increasing interest in considering the problem of estimation in systems with sensor gain degradation, which has been addressed by various approaches assuming knowledge of the state-space model. For example, He, Wang \& Zhou (2009) derived a robust $H_{\infty}$ filtering algorithm for a class of nonlinear time-varying system with parameter uncertainties and probabilistic sensor faults; Liu, Wang \& Zhou (2014) studied the optimal filtering problem for networked time-varying systems with stochastic gain degradations by a recursive matrix equation approach; and Liu, Wang, He \& Zhou (2016) obtained a minimum variance filtering algorithm for a class of time-varying systems.

As commented above, there may also be failures in transmitting the measurements, provoking random delays in data arrival, which are usually modelled by Bernoulli random variables. Assuming that these variables are independent, previous studies have addressed the estimation problem in two ways, either assuming that the state-space model is completely known, or using the information provided by the covariance functions of the process involved in the observation model. Under both approaches, recursive filtering algorithms have been derived for the case of one-step random delays (see e.g. Caballero-Águila, Hermoso-Carazo, Jiménez-López, Linares-Pérez \& Nakamori, 
2010; Hounkpevi \& Yaz, 2007) and generalised to that of measurements subject to bounded multiple-step random delays (see e.g. Caballero-Águila, Hermoso-Carazo \& Linares-Pérez, 2017b; Li, Sun \& Ma, 2014). Nevertheless, in real-world communication systems, current delays are usually correlated with the previous ones, and a reasonable way to model the dependence is to use homogeneous Markov chains. As in the case of delays modelled by independent random variables, estimation algorithms from Markovian delayed observations have also been deduced by assuming that the statespace model is known and using covariance information. Assuming that the state-space model is known, optimal estimation problems in networked systems with correlated transmission delays are discussed in Han \& Zhang (2009) and Han, Zhang \& Fu (2013), where the delay process is modelled by a two-state Markov chain and by a multi-state Markov chain, respectively. In this respect, too, Song, Yu \& Zhang (2009) investigated the $H_{\infty}$ filtering problem in a class of network systems with random delays modelled by a Markov chain. Also, using only covariance information and modelling the delays by Markov chains, recursive filtering and smoothing algorithms to estimate a signal from one-step delayed observations were proposed by García-Ligero, Hermoso-Carazo \& Linares-Pérez (2015) and the filtering problem has been recently investigated by García-Ligero, Hermoso-Carazo \& Linares-Pérez (2018), assuming that the measurements can be randomly delayed by one or two sampling times.

In most estimation algorithms, it is usually assumed that the additive noises that affect the dynamic system are white and uncorrelated. However, this hypothesis may not be valid in many real problems, in which these noises are often correlated. This consideration has aroused great interest, motivating researchers to study the estimation problem in systems with correlated and/or cross-correlated noises. For example, when the state-space model is known, assuming that the signal process and measurement noises are one-step autocorrelated and two-step cross-correlated, filtering algorithms have been derived for uncertain systems (see e.g. Feng, Wang \& Zeng, 2013; Tian, Sun $\& \mathrm{Li}, 2016)$. This problem has been also addressed for stochastic systems with random parameter matrices (see e.g. Caballero-Águila, Hermoso-Carazo, Linares-Pérez \& Wang, 2019; Hu, Wang \& Gao, 2013; Sun, Tian \& Lin, 2017). Recently, assuming that the signal process and the observation noises are $N$-step auto-and cross-correlated, the estimation problem has been investigated in Tian, Sun \& Lin (2019). On the other hand, assuming that the evolution model generating the signal process is not available, filtering algorithms have been also derived when the measured outputs are perturbed by correlated noises in Caballero-Águila Hermoso-Carazo \& Linares-Pérez. (2017c), considering multiple random transmission uncertainties, and in García-Ligero, Hermoso-Carazo \& Linares-Pérez (2017) for the case of multiple packet dropouts.

In view of the above considerations, our aim in the present study is to investigate the estimation problem for systems with stochastic sensor gain degradation and correlated measurement noise, subject to one or two-step random delays during the transmission. Specifically, we assume that the sensor gain degradation is represented by a white sequence of random variables with values in $[0,1]$ reflecting partial deterioration of the signal; this assumption clearly covers the missing measurement problem as a special case. Furthermore, the measurement noise is correlated at consecutive sampling times. The delays are modelled by a homogeneous discrete-time Markov chain with three states, which provides a reasonable description of a possible correlation of delays at different sampling times. Assuming no signal equation is available, the estimators are derived using the first and second-order moments of the signal and the processes 
involved in the observation model. The least-squares linear prediction, filtering and fixed-smoothing algorithms are obtained by an innovation approach which enables the estimation algorithms to be derived straightforwardly.

Therefore, as in García-Ligero et al. (2018), we assume that the processed measurements can be one or two-steps delayed, and that the delays are modelled by a homogeneous Markov chain with three states. In addition to addressing the fixedpoint smoothing, and not just the filtering problem, the main novelty of the present research with regard to the earlier work, is that we now consider a more general model of measurement outputs. In this paper, the outputs are affected by gain degradations and correlated additive noise, whereas in García-Ligero et al. (2018), the outputs were only perturbed by additive white noise. Since the presence of degradations and the correlation of the measurement noise can strongly influence the performance of estimators, the filtering algorithm in the previous paper cannot be applied to the model proposed in this paper. The generality of this new model extends the scope of the proposed algorithms, which can be applied not only to models such as the one considered in García-Ligero et al. (2018) but also to those with missing measurements.

The main contributions of this paper can be summarised as follows: (1) The model considered is affected by two uncertainties; namely, sensor gain degradation and random delays during the transmission, being the delays modelled by a three-state homogeneous Markov chain. This model is more general than those in which the uncertainties are described by independent Bernoulli random variables and, therefore, provides greater applicability. (2) Unlike other approaches, measurement noise is one-step correlated. (3) The proposed estimators, predictor, filter and fixed-point smoother, based on covariance information, do not require knowledge of the evolution model generating the signal, with the added advantage that they may also be applicable to the conventional state-space model. (4) The proposed estimation algorithms are derived by an innovation approach, which facilitates their derivation, since the innovation is a white process.

The rest of this paper is organised in the following way. The next section describes the model at hand and details the assumptions under which the estimation problem is addressed. In Section 3, the least-squares linear estimation problem is formulated, using an innovation approach, and the one-stage observation predictor is obtained. The recursive prediction, filtering and fixed-point smoothing algorithms and their error covariance matrices, which provide a measure of the estimators performance, are then derived in Section 4. A simulation example illustrating the performance of the proposed estimators is given in Section 5, after which some concluding remarks and future research are made.

Notation. The notation used throughout this paper is standard. $\mathbb{R}^{n \times m}$ denotes the set of all $n \times m$ matrices. If the dimensions of vectors or matrices are not explicitly stated, they are assumed to be compatible with algebraic operations. $\left(C_{1}|\ldots| C_{n}\right)$ denotes a matrix partitioned into submatrices $C_{1}, \ldots, C_{n}$ and $I_{n}$ is $n \times n$ identity matrix. $\otimes$ represents the Kronecker product, $\delta_{k, s}$ denotes the Kronecker delta function and $c \wedge d$ and $c \vee d$ represent, respectively, the minimum and maximum of $c$ and $d$, for any $c, d \in \mathbb{R}$. For any function $F_{k, j}$ depending on the time instants $k$ and $j$, we will write $F_{k}=F_{k, k}$; analogously, $L^{d}=L^{d d}$ will be written for any function $L^{d e}$. 


\section{Model description}

In signal processing, it is usually assumed that the sensor gain is randomly degraded, and, therefore, partially deteriorated measurements may be obtained. In this context, let us consider an $n$-dimensional signal, $x_{k}$, whose scalar measured outputs, $z_{k}$, are described by

$$
z_{k}=\gamma_{k} H_{k} x_{k}+v_{k}, \quad k \geq 1,
$$

where, for all $k \geq 1$, the random variable $\gamma_{k}$ accounts for sensor gain degradation, $H_{k}$ is a known matrix and $v_{k}$ is the measurement noise.

Our aim is to obtain the least-squares (LS) linear predictor, filter and fixed-point smoother of the signal. These estimators are derived by a covariance-based approach; that is, we assume that the signal evolution model is unknown and only their first and second-order moments are known; specifically:

Assumption 1. The signal process, $\left\{x_{k}, k \geq 1\right\}$, has zero mean and its autocovariance function is expressed in a separable form as follows,

$$
E\left[x_{k} x_{h}^{T}\right]=A_{k} B_{h}^{T}, \quad h \leq k,
$$

where, $A_{k}, B_{h} \in \mathbb{R}^{n \times M}$ are known matrices.

Remark 1. Note that the required separable form on the signal autocovariance function is not very restrictive. In fact, this assumption covers situations in which the signal evolution model is known, for both stationary and non-stationary signals. For example, for the state-space model with stationary signals, $x_{k}=\Phi x_{k-1}+w_{k-1}$, the covariance function can be expressed as $E\left[x_{k} x_{h}^{T}\right]=\Phi^{k-h} E\left[x_{h} x_{h}^{T}\right], h \leq k$, and Assumption 1 is satisfied taking $A_{k}=\Phi^{k}$ and $B_{h}=E\left[x_{h} x_{h}^{T}\right]\left(\Phi^{-h}\right)^{T}$. If non-stationary signals, $x_{k}=\Phi_{k} x_{k-1}+w_{k-1}$, are considered, the covariance function can be expressed as $E\left[x_{k} x_{h}^{T}\right]=\Phi_{k, h} E\left[x_{h} x_{h}^{T}\right], h \leq k$, with $\Phi_{k, h}=\Phi_{k} \cdots \Phi_{h+1}$; then, taking $A_{k}=\Phi_{k, 0}$ and $B_{h}=E\left[x_{h} x_{h}^{T}\right]\left(\Phi_{h, 0}^{-1}\right)^{T}$, Assumption 1 is clearly satisfied. Hence the separability hypothesis of the covariance function provides a unified approach to address different situations based on the state-space model and it is not necessary to obtain specific algorithms for each case. Furthermore, we highlight that, although a state-space model can be generated from covariances, when only this kind of information is available, it is preferable to address the estimation problem directly using covariances, thus obviating the need for prior identification of the state-space model.

Assumption 2. The multiplicative process, $\left\{\gamma_{k}, k \geq 1\right\}$, is a white sequence of scalar variables taking values in the interval $[0,1]$, with known means and variances, $E\left[\gamma_{k}\right]=$ $\bar{\gamma}_{k}$ and $\operatorname{Var}\left[\gamma_{k}\right]=\sigma_{k}^{2}$.

This assumption on the random variables $\gamma_{k}$ is more precise for quantifying the sensor gain degradation than that traditionally used where the random variables only take the values zero and one.

Assumption 3. The measurement noise, $\left\{v_{k}, k \geq 1\right\}$, is a zero-mean one-step correlated scalar process with $E\left[v_{k} v_{h}\right]=R_{k} \delta_{k, h}+R_{k, k-1} \delta_{k-1, h}, \quad h \leq k$.

During the transmission of the measurements to the processing centre, delays com- 
monly occur, due, for example, to congestion in the communication channel. In this paper, the absence or presence of delays in the transmission, and their magnitude, is modelled by random variables $\left\{\theta_{k}, k \geq 1\right\}$, that take values in $S=\{0,1,2\}$, describing whether the measures arrive on time or they are delayed by one or two sampling times. Specifically, if $\theta_{k}=a, a=1,2$, this means that the $k$-th measurement is delayed by $a$ sampling periods; otherwise, if $\theta_{k}=0$, there is no delay in arrival. This situation is modelled as in García-Ligero et al. (2018); that is, the received measurements, which are denoted by $y_{k}, k \geq 1$, representing the observation process, are described as:

$$
y_{k}=\sum_{a=0}^{(k-1) \wedge 2} \delta_{\theta_{k}, a} z_{k-a}, \quad k \geq 1,
$$

where the following assumption on the random variables $\theta_{k}$ modelling the delays is taken on:

Assumption 4. $\left\{\theta_{k}, k \geq 1\right\}$ is a homogeneous Markov chain taking values in $S=$ $\{0,1,2\}$, with known initial distribution $\pi_{a}^{(1)}=P\left(\theta_{1}=a\right), a \in S$, and transition probability matrix $\mathbf{P}=\left(p_{a b}\right)_{a, b \in S}$, where $p_{a b}=P\left(\theta_{k+1}=b / \theta_{k}=a\right), \quad a, b \in S$.

Finally, the following hypothesis about the signal and processes involved in the measurement model is assumed in the derivation of the LS linear estimators.

Assumption 5. The signal, $\left\{x_{k}, k \geq 1\right\}$, and the processes $\left\{\gamma_{k}, k \geq 1\right\},\left\{v_{k}, k \geq 1\right\}$ and $\left\{\theta_{k}, k \geq 1\right\}$, are mutually independent.

In García-Ligero et al. (2018), the measurement outputs are only affected by an additive white noise; in the current paper a more general model is considered, since the measurement outputs are affected by gain degradations and, in addition, the additive noise is correlated, a situation that arises in many practical problems. Although in both models the processed measurements can be delayed and the delays are modelled in the same way, by a homogeneous Markov chain, the algorithm obtained in GarcíaLigero et al. (2018) cannot be applied to the model at hand since the presence of gain degradations on measurements and the correlation of measurement noise exert a strong influence on the performance of the estimators. Therefore, our aim is to derive new estimation algorithms, prediction, filtering and fixed-point smoothing, for the model (1)-(2). Furthermore, the algorithms that we propose can be applied to other models, such as the one considered in García-Ligero et al. (2018), as well as to the model of missing measurements.

\section{Least-squares linear estimation problem by innovation approach}

\subsection{Innovation approach}

The LS linear estimation of the signal, $x_{k}$, from randomly delayed measurements up to time $L, y_{1}, \cdots, y_{L}$, is addressed using an innovation approach. This approach is based on an orthogonalisation procedure by means of which the observation process $\left\{y_{k} ; k \geq 1\right\}$ is transformed into an equivalent one, called innovation process; this new process is denoted by $\left\{\mu_{k} ; k \geq 1\right\}$ and defined as $\mu_{k}=y_{k}-\widehat{y}_{k / k-1}$, where $\widehat{y}_{k / k-1}$ is the LS linear estimator of $y_{k}$ from the previous observations, $y_{1}, \ldots, y_{k-1}$. Linear estimation is then performed by replacing the observation process with the 
innovation one, since both processes provide the same information. Therefore, the LS linear estimator of a random vector $u_{k}$ based on the observations $\left\{y_{1}, \ldots, y_{L}\right\}$, denoted by $\widehat{u}_{k / L}$, is expressed as a linear combination of the innovations $\left\{\mu_{1}, \ldots, \mu_{L}\right\}$; specifically:

$$
\widehat{u}_{k / L}=\sum_{h=1}^{L} E\left[u_{k} \mu_{h}\right] \Sigma_{\mu_{h}}^{-1} \mu_{h}, \quad k \geq 1,
$$

where $\Sigma_{\mu_{h}}=E\left[\mu_{h}^{2}\right]$ denotes the innovation variance.

The advantage of using this approach is that the estimator expressed as a linear combination of innovations provides a simpler form of obtaining estimation algorithms than that obtained when it is expressed as a linear combination of observations, since the innovation process is white.

In accordance with expression (3), the first step to obtain the signal estimators is to calculate an explicit formula for the innovation, $\mu_{k}=y_{k}-\widehat{y}_{k / k-1}$, or, equivalently, for the one-stage observation predictor, $\widehat{y}_{k / k-1}$; this process is described in the following section.

\subsection{One-stage observation predictor}

From (3), the one-stage observation predictor is given by

$$
\widehat{y}_{k / k-1}=\sum_{h=1}^{k-1} \mathcal{Y}_{k, h} \Sigma_{\mu_{h}}^{-1} \mu_{h}, \quad k \geq 2 ; \quad \widehat{y}_{1 / 0}=0,
$$

where $\mathcal{Y}_{k, h}=E\left[y_{k} \mu_{h}\right]=E\left[y_{k} y_{h}\right]-E\left[y_{k} \widehat{y}_{h / h-1}\right]$. Then, in order to derive an explicit expression of the one-stage observation predictor it is necessary to determine these coefficients, $\mathcal{Y}_{k, h}, 1 \leq h \leq k-1$. The following results provide the expression required.

Lemma 3.1. Under the model assumptions, the observation process given by (2) verifies that

$$
E\left[y_{k} y_{h}\right]=\mathcal{A}_{k} \mathcal{B}_{h}^{T}+\mathcal{F}_{k, h}, \quad 1 \leq h \leq k-1,
$$

where:

i) $\mathcal{A}_{k}=\left(\bar{\gamma}_{k} H_{k} A_{k}\left|\bar{\gamma}_{k-1} H_{k-1} A_{k-1}\right| \bar{\gamma}_{k-2} H_{k-2} A_{k-2}\right)\left(\mathbf{P}^{k} \otimes I_{M}\right)^{T}, \quad k \geq 1$,

$\mathcal{B}_{k}=\left(\bar{\gamma}_{k} H_{k} B_{k}\left|\bar{\gamma}_{k-1} H_{k-1} B_{k-1}\right| \bar{\gamma}_{k-2} H_{k-2} B_{k-2}\right) \Pi^{(k)}\left(\mathbf{P}^{-k} \otimes I_{M}\right), \quad k \geq 1$,

being $\Pi^{(k)}=\operatorname{diag}\left(\pi_{0}^{(k)}, \pi_{1}^{(k)}, \pi_{2}^{(k)}\right) \otimes I_{M}$, with $\pi_{a}^{(k)}=P\left(\theta_{k}=a\right), a \in S$, and $\bar{\gamma}_{h} H_{h} C_{h}$ equal to zero for $C_{h}=A_{h}, B_{h}, h=-1,0$.

ii) $\mathcal{F}_{k, h}=0, \quad h<k-3$, 


$$
\begin{aligned}
\mathcal{F}_{k, k-3}= & \pi_{0}^{(k-3)} p_{02}^{(3)} R_{k-2, k-3}, \\
\mathcal{F}_{k, k-2}= & \pi_{0}^{(k-2)} p_{02}^{(2)} \sigma_{k-2}^{2} H_{k-2} A_{k-2} B_{k-2}^{T} H_{k-2}^{T} \\
& +\sum_{a=1}^{(k-1) \wedge 2} \sum_{b=0}^{(k-3) \wedge(a-1)} \pi_{b}^{(k-2)} p_{b a}^{(2)} R_{k-a, k-2-b}, \\
\mathcal{F}_{k, k-1}= & \pi_{0}^{(k-1)} p_{02}^{(1)} \bar{\gamma}_{k-2} \bar{\gamma}_{k-1} H_{k-2}\left(B_{k-2} A_{k-1}^{T}-A_{k-2} B_{k-1}^{T}\right) H_{k-1}^{T}\left(1-\delta_{k, 2}\right) \\
& +\sum_{a=1}^{(k-1) \wedge 2} \pi_{a-1}^{(k-1)} p_{a-1 a}^{(1)} \sigma_{k-a}^{2} H_{k-a} A_{k-a} B_{k-a}^{T} H_{k-a}^{T} \\
& +\sum_{a=0}^{(k-1) \wedge 2} \sum_{b=0}^{(k-2) \wedge a} \pi_{b}^{(k-1)} p_{b a}^{(1)} R_{k-a, k-1-b} .
\end{aligned}
$$

Proof. $p_{a b}^{(k)}=P\left(\theta_{h+k}=b / \theta_{h}=a\right), \quad h, k \geq 1, a, b \in S\left(p_{a b}^{(1)}=p_{a b}\right)$, denotes the $k$-step transition probability from state $a$ to state $b$, and so it is clear that

$$
E\left[\delta_{\theta_{k}, a} \delta_{\theta_{h}, b}\right]=P\left(\theta_{k}=a / \theta_{h}=b\right) P\left(\theta_{h}=b\right)=p_{b a}^{(k-h)} \pi_{b}^{(h)}, \quad h<k .
$$

Then, from the independence assumption, the properties of the process $\left\{\gamma_{k}, k \geq 1\right\}$ and the separability of the signal autocovariance function, $E\left[y_{k} y_{h}\right]$ is expressed as

$$
\begin{aligned}
E\left[y_{k} y_{h}\right]= & \sum_{a=0}^{(k-1) \wedge 2} \sum_{b=0}^{(h-1) \wedge 2} \pi_{b}^{(h)} p_{b a}^{(k-h)} \bar{\gamma}_{k-a} \bar{\gamma}_{h-b} H_{k-a} E\left[x_{k-a} x_{h-b}^{T}\right] H_{k-b}^{T} \\
& +\sum_{a=0}^{(k-1) \wedge 2} \sum_{b=0}^{(h-1) \wedge 2} \pi_{b}^{(h)} p_{b a}^{(k-h)} \sigma_{k-a}^{2} H_{k-a} A_{k-a} B_{k-a}^{T} H_{k-a}^{T} \delta_{k-a, h-b} \\
& +\sum_{a=0}^{(k-1) \wedge 2} \sum_{b=0}^{(h-1) \wedge 2} \pi_{b}^{(h)} p_{b a}^{(k-h)} E\left[v_{k-a} v_{h-b}\right], \quad h \leq k-1 .
\end{aligned}
$$

Again, using the separability assumption, the first addend of the second term of the above expression is expressed as

$$
\mathcal{A}_{k} \mathcal{B}_{h}^{T}+\pi_{0}^{(k-1)} p_{02}^{(1)} \bar{\gamma}_{k-2} \bar{\gamma}_{k-1} H_{k-2}\left(B_{k-2} A_{k-1}^{T}-A_{k-2} B_{k-1}^{T}\right) H_{k-1}^{T} \delta_{k-1, h} .
$$

The second addend is reduced to the cases in which $b=a-(k-h)$; then, $a \geq k-h$, and hence

$$
\sum_{a=k-h}^{(k-1) \wedge 2} \pi_{a-(k-h)}^{(h)} p_{a-(k-h) a}^{(k-h)} \sigma_{k-a}^{2} H_{k-a} A_{k-a} B_{k-a}^{T} H_{k-a}^{T} .
$$

Finally, taking into account the one-step correlated noise assumption, the third addend is null except when $k-a-1 \leq h-b \leq k-a+1$; that is, for $h=k-3, k-2, k-1$. 
From above considerations, (5) for $E\left[y_{k} y_{h}\right], 1 \leq h \leq k-1$, is obtained.

Lemma 3.2. Under the model assumptions, the coefficients $\mathcal{Y}_{k, h}=E\left[y_{k} \mu_{h}\right]$ are given by

$$
\mathcal{Y}_{k, h}=\mathcal{A}_{k} J_{h}^{y}+\mathcal{G}_{k, h}, \quad 1 \leq h \leq k-1
$$

where

$$
J_{h}^{y}=\mathcal{B}_{h}^{T}-\sum_{j=1}^{h-1} J_{j}^{y} \Sigma_{\mu_{j}}^{-1} \mathcal{Y}_{h, j}, \quad h \geq 2 ; \quad J_{1}^{y}=\mathcal{B}_{1}^{T},
$$

and

$$
\mathcal{G}_{k, h}= \begin{cases}0, & h<k-3, \\ \mathcal{F}_{k, h}, & h=1 \text { or } h=k-3, \\ \mathcal{F}_{k, h}-\sum_{j=(k-3) \vee 1}^{h-1} \mathcal{G}_{k, j} \Sigma_{\mu_{j}}^{-1}\left(\mathcal{A}_{h} J_{j}^{y}+\mathcal{G}_{h, j}\right), & h \geq 2, h=k-2, k-1 .\end{cases}
$$

Proof. Taking into account that $\mu_{k}=y_{k}-\widehat{y}_{k / k-1}$, expression (4) for the one-step observation predictor and (5), the coefficients, $\mathcal{Y}_{k, h}=E\left[y_{k} y_{h}\right]-E\left[y_{k} \widehat{y}_{h / h-1}\right], 1 \leq h \leq$ $k-1$, are expressed as:

$$
\mathcal{Y}_{k, h}= \begin{cases}\mathcal{A}_{k} \mathcal{B}_{1}^{T}+\mathcal{F}_{k, 1}, & h=1 ; \\ \mathcal{A}_{k} \mathcal{B}_{h}^{T}+\mathcal{F}_{k, h}-\sum_{j=1}^{h-1} \mathcal{Y}_{k, j} \Sigma_{\mu_{j}}^{-1} \mathcal{Y}_{h, j}, & 2 \leq h \leq k-1 .\end{cases}
$$

From (7), expression (6) for the coefficients $\mathcal{Y}_{k, h}$ is evident, by defining $\mathcal{G}_{k, 1}=\mathcal{F}_{k, 1}$ and $\mathcal{G}_{k, h}=\mathcal{F}_{k, h}-\sum_{j=1}^{h-1} \mathcal{G}_{k, j} \Sigma_{\mu_{j}}^{-1} \mathcal{Y}_{h, j}, h \geq 2$.

Finally, expression (8) for $\mathcal{G}_{k, h}, h \geq 2$, is obtained using (6) for $\mathcal{Y}_{k, h}$, taking into account that $\mathcal{G}_{k, h}$ is a linear function of $\mathcal{F}_{k, j}, j=1, \ldots, h$, and $\mathcal{F}_{k, j}=0, j<k-3$, as it was established in Lemma 3.1.

Now, substituting expression (6) of the coefficients $\mathcal{Y}_{k, h}$ in (4) and defining $O_{k}^{y}=$ $\sum_{h=1}^{k} J_{h}^{y} \Sigma_{\mu_{h}}^{-1} \mu_{h}, k \geq 1$, and $O_{0}^{y}=0$, the one-stage observation predictor is given by

$$
\widehat{y}_{k / k-1}=\mathcal{A}_{k} O_{k-1}^{y}+\sum_{h=(k-3) \vee 1}^{k-1} \mathcal{G}_{k, h} \Sigma_{\mu_{h}}^{-1} \mu_{h}, \quad k \geq 2 ; \quad \widehat{y}_{1 / 0}=0 .
$$

Once the expression of the one-stage observation predictor expression is determined, we can obtain the estimation algorithms, which is purpose of the next section. 


\section{Recursive prediction, filtering and fixed point smoothing algorithms}

This section considers the problem of obtaining recursive algorithms for the predictors, filter (Theorem 4.1) and fixed-point smoothers (Theorem 4.2) of the signal. Expressions for their respective estimation error covariance matrices are also deduced, and these will be used in Section 5 as a measure of the accuracy of the proposed estimators.

Theorem 4.1. Under the model assumptions, the predictors and filter, $\widehat{x}_{k / L}, L \leq k$, of the signal, $x_{k}$, are obtained by

$$
\widehat{x}_{k / L}=A_{k} O_{L}^{x}, \quad L \leq k,
$$

and their prediction and filtering error covariance matrices, $\Sigma_{k / L}=E\left[\left(x_{k}-\widehat{x}_{k / L}\right)\left(x_{k}-\right.\right.$ $\left.\widehat{x}_{k / L}\right)^{T}$, are given by

$$
\Sigma_{k / L}=A_{k}\left(B_{k}^{T}-r_{L}^{x} A_{k}^{T}\right), \quad L \leq k .
$$

The innovation, $\mu_{L}$, and its variance, $\Sigma_{\mu_{L}}$, are obtained by

$$
\begin{aligned}
\mu_{L}= & y_{L}-\mathcal{A}_{L} O_{L-1}^{y}-\sum_{h=(L-3) \vee 1}^{L-1} \mathcal{G}_{L, h} \Sigma_{\mu_{h}}^{-1} \mu_{h}, \quad L \geq 2 ; \quad \mu_{1}=y_{1}, \\
\Sigma_{\mu_{L}}= & \sum_{a=0}^{(L-1) \wedge 2} \pi_{a}^{(L)}\left(\sigma_{L-a}^{2} H_{L-a} A_{L-a} B_{L-a}^{T} H_{L-a}^{T}+R_{L-a}\right)+\mathcal{A}_{L} J_{L}^{y} \\
& -\sum_{j=(L-3) \vee 1}^{L-1} \mathcal{G}_{L, j} \Sigma_{\mu_{j}}^{-1}\left(\mathcal{A}_{L} J_{j}^{y}+\mathcal{G}_{L, j}\right)^{T}, \quad L \geq 2 ; \\
\Sigma_{\mu_{1}}= & \mathcal{A}_{1} \mathcal{B}_{1}^{T}+\pi_{0}^{(1)}\left(\sigma_{1}^{2} H_{1} A_{1} B_{1}^{T} H_{1}^{T}+R_{1}\right) .
\end{aligned}
$$

The vectors $O_{L}^{d}, d=x, y$, and the matrices $r_{L}^{d e}, d, e=x, y$, are recursively calculated by

$$
\begin{aligned}
& O_{L}^{d}=O_{L-1}^{d}+J_{L}^{d} \Sigma_{\mu_{L}}^{-1} \mu_{L}, \quad L \geq 1 ; \quad O_{0}^{d}=0, \\
& r_{L}^{d e}=r_{L-1}^{d e}+J_{L}^{d} \Sigma_{\mu_{L}}^{-1} J_{L}^{e T}, \quad L \geq 1 ; \quad r_{0}^{d e}=0 .
\end{aligned}
$$

with

$$
\begin{aligned}
J_{L}^{x} & =\sum_{a=0}^{(L-1) \wedge 2} \pi_{a}^{(L)} \bar{\gamma}_{L-a} B_{L-a}^{T} H_{h-a}^{T}-r_{L-1}^{x y} \mathcal{A}_{L}^{T}-\sum_{j=(L-3) \vee 1}^{L-1} J_{j}^{x} \Sigma_{\mu_{j}}^{-1} \mathcal{G}_{L, j}, \quad L \geq 2 ; \\
J_{1}^{x} & =\pi_{0}^{(1)} \bar{\gamma}_{1} B_{1}^{T} H_{1}^{T},
\end{aligned}
$$




$$
J_{L}^{y}=\mathcal{B}_{L}^{T}-r_{L-1}^{y} \mathcal{A}_{L}^{T}+\sum_{j=(L-3) \vee 1}^{L-1} J_{j}^{y} \Sigma_{\mu_{j}}^{-1} \mathcal{G}_{L, j}, \quad L \geq 2 ; \quad J_{1}^{y}=\mathcal{B}_{1}^{T}
$$

Proof. Taking into account the general expression of estimator (3), to derive the LS linear estimators, $\widehat{x}_{k / L}, L \leq k$, it is necessary to calculate the coefficients

$$
\mathcal{X}_{k, h}=E\left[x_{k} \mu_{h}\right]=E\left[x_{k} y_{h}\right]-E\left[x_{k} \widehat{y}_{h / h-1}\right], \quad h \leq k .
$$

From (1)-(2), taking into account the model hypotheses and that, for $h-a \leq k$, $E\left[x_{k} z_{h-a}\right]=\bar{\gamma}_{h-a} A_{k} B_{h-a}^{T} H_{h-a}^{T}$, we obtain

$$
E\left[x_{k} y_{h}\right]=\sum_{a=0}^{(h-1) \wedge 2} \pi_{a}^{(h)} \bar{\gamma}_{h-a} A_{k} B_{h-a}^{T} H_{h-a}^{T}, \quad h \leq k ;
$$

then, substituting $\widehat{y}_{h / h-1}=\sum_{j=1}^{h-1} \mathcal{Y}_{h, j} \Sigma_{\mu_{j}}^{-1} \mu_{j}$ and $\widehat{y}_{1 / 0}=0$ in $E\left[x_{k} \widehat{y}_{h / h-1}\right]$, it follows straightforwardly that the coefficients are expressed as

$$
\begin{aligned}
& \mathcal{X}_{k, h}=A_{k} \sum_{a=0}^{(h-1) \wedge 2} \pi_{a}^{(h)} \bar{\gamma}_{h-a} B_{h-a}^{T} H_{h-a}^{T}-\sum_{j=1}^{h-1} \mathcal{X}_{k, j} \Sigma_{\mu_{j}}^{-1} \mathcal{Y}_{h, j}, \quad h \geq 2 ; \\
& \mathcal{X}_{k, 1}=A_{k} \pi_{0}^{(1)} \bar{\gamma}_{1} B_{1}^{T} H_{1}^{T},
\end{aligned}
$$

or equivalently as

$$
\mathcal{X}_{k, h}=A_{k} J_{h}^{x}, \quad 1 \leq h \leq k,
$$

where $J_{h}^{x}$ is a function satisfying

$$
\begin{aligned}
J_{h}^{x} & =\sum_{a=0}^{(h-1) \wedge 2} \pi_{a}^{(h)} \bar{\gamma}_{h-a} B_{h-a}^{T} H_{h-a}^{T}-\sum_{j=1}^{h-1} J_{j}^{x} \Sigma_{\mu_{j}}^{-1} \mathcal{Y}_{h, j}, \quad h \geq 2 ; \\
J_{1}^{x} & =\pi_{0}^{(1)} \bar{\gamma}_{1} B_{1}^{T} H_{1}^{T} .
\end{aligned}
$$

Now, substituting (18) in $\widehat{x}_{k / L}=\sum_{h=1}^{L} \mathcal{X}_{k, h} \Sigma_{\mu_{h}}^{-1} \mu_{h}, L \leq k$, and defining $O_{L}^{x}=$ $\sum_{h=1}^{L} J_{h}^{x} \Pi_{h}^{-1} \mu_{h}, L \geq 1$, and $O_{0}^{x}=0$, expression (10) is deduced.

Formula (11) for $\Sigma_{k / L}=E\left[\left(x_{k}-\widehat{x}_{k / L}\right)\left(x_{k}-\widehat{x}_{k / L}\right)^{T}\right]=E\left[x_{k} x_{k}^{T}\right]-E\left[\widehat{x}_{k / L} \widehat{x}_{k / L}^{T}\right]$ is derived immediately from (10) and Assumption 1, by defining $r_{L}^{x}=E\left[O_{L}^{x} O_{L}^{x T}\right]$.

Expression (12) for the innovation is obtained directly from (9). To prove (13) we first apply the Orthogonal Projection Lemma to express $\Sigma_{\mu_{L}}=E\left[\left(y_{L}\right)^{2}\right]-E\left[y_{L} \widehat{y}_{L / L-1}\right]$, and both expectations are calculated as follows. From (1)-(2) and the model hypothe- 
ses, it is clear that

$$
E\left[\left(y_{L}\right)^{2}\right]=\mathcal{A}_{L} \mathcal{B}_{L}^{T}+\sum_{a=0}^{(L-1) \wedge 2} \pi_{a}^{(L)}\left(\sigma_{L-a}^{2} H_{L-a} A_{L-a} B_{L-a}^{T} H_{L-a}^{T}+R_{L-a}\right), L \geq 1,
$$

and using (4) for the observation predictor, (6) for $\mathcal{Y}_{L, h}$, and (7) for $\sum_{h=1}^{L-1} J_{h}^{y} \Sigma_{\mu_{h}}^{-1} \mathcal{Y}_{L, h}$, we deduce

$$
\begin{aligned}
& E\left[y_{L} \widehat{y}_{L / L-1}\right]=\mathcal{A}_{L}\left(\mathcal{B}_{L}^{T}-J_{L}^{y}\right)+\sum_{h=(L-3) \vee 1}^{L-1} \mathcal{G}_{L, h} \Sigma_{\mu_{h}}^{-1}\left(\mathcal{A}_{L} J_{h}^{y}+\mathcal{G}_{L, h}\right)^{T}, \quad L \geq 2 ; \\
& E\left[y_{1} \widehat{y}_{1 / 0}\right]=0 .
\end{aligned}
$$

Then, from both expectations, expression (13) is obtained.

The recursive relations (14) and (15) follow immediately from the definitions $O_{L}^{d}=$ $\sum_{h=1}^{L} J_{h}^{d} \Sigma_{\mu_{h}}^{-1} \mu_{h}, d=x, y$, and $r_{L}^{d e}=E\left[O_{L}^{d} O_{L}^{e T}\right]=\sum_{h=1}^{L} J_{h}^{d} \Sigma_{\mu_{h}}^{-1} J_{h}^{e T}, d, e=x, y$, respectively.

Expression (16) for $J_{L}^{x}$ is obtained substituting (6) into (19) for $h=L$ and using the definition of $r_{L}^{x y}$. By an analogous reasoning, but now using the definition of $r_{L}^{y}$, expression (17) for $J_{L}^{y}$ is derived.

In the following theorem, LS linear estimators of the signal, $x_{k}$, are obtained when the available observations are $y_{1}, \ldots, y_{k+s}$, with $s \geq 1$; these estimators are derived from a recursive fixed-point smoothing algorithm.

Theorem 4.2. Under the model assumptions, the fixed-point smoothers are recursively obtained by

$$
\widehat{x}_{k / k+s}=\widehat{x}_{k / k+s-1}+\mathcal{X}_{k, k+s} \Sigma_{\mu_{k+s}}^{-1} \mu_{k+s}, \quad s \geq 1,
$$

and the fixed-point smoothing error covariance matrices, $\Sigma_{k / k+s}$, satisfy

$$
\Sigma_{k / k+s}=\Sigma_{k / k+s-1}-\mathcal{X}_{k, k+s} \Sigma_{\mu_{k+s}}^{-1} \mathcal{X}_{k, k+s}^{T}, \quad s \geq 1
$$

their initial conditions, $\widehat{x}_{k / k}$ and $\Sigma_{k / k}$, are given in Theorem 4.1. 
The coefficients $\mathcal{X}_{k, k+s}=E\left[x_{k} \mu_{k+s}\right]$ are given by

$$
\begin{aligned}
\mathcal{X}_{k, k+1}= & \pi_{0}^{(k+1)} \bar{\gamma}_{k+1} B_{k} A_{k+1}^{T} H_{k+1}^{T}+A_{k} \sum_{a=1}^{k \wedge 2} \pi_{a}^{(k+1)} \bar{\gamma}_{k+1-a} B_{k+1-a}^{T} H_{k+1-a}^{T} \\
& -\mathcal{E}_{k} \mathcal{A}_{k+1}^{T}-\sum_{h=(k-2) \vee 1}^{k} \mathcal{X}_{k, h} \Sigma_{\mu_{h}}^{-1} \mathcal{G}_{k+1, h}, \\
\mathcal{X}_{k, k+s}= & B_{k} \sum_{a=0}^{2} \pi_{0}^{(k+s)} \bar{\gamma}_{k+s-a} A_{k+s-a}^{T} H_{k+s-a}^{T}-\mathcal{E}_{k, k+s-1} \mathcal{A}_{k+s}^{T} \\
& -\sum_{h=(k+s-3) \vee 1}^{k+s-1} \mathcal{X}_{k, h} \Sigma_{\mu_{h}}^{-1} \mathcal{G}_{k+s, h}, \quad s \geq 2,
\end{aligned}
$$

and $\mathcal{E}_{k, k+s}=E\left[\widehat{x}_{k / k+s} O_{k+s}^{y T}\right]$ satisfy the following recursive formula

$$
\mathcal{E}_{k, k+s}=\mathcal{E}_{k, k+s-1}+\mathcal{X}_{k, k+s} \Sigma_{\mu_{k+s}}^{-1} J_{k+s}^{y T}, \quad s \geq 1 ; \quad \mathcal{E}_{k}=A_{k} r_{k}^{x y}
$$

Proof. Recursive relation (20) for the smoothers follows immediately from (3). Moreover, from (20) and the uncorrelation of $\mu_{k+s}$ and $\widehat{x}_{k / k+s-1}$, expression (21) is immediately obtained.

In order to determine relation (22) for $\mathcal{X}_{k, k+s}=E\left[x_{k} y_{k+s}\right]-E\left[x_{k} \widehat{y}_{k+s / k+s-1}\right], s \geq 1$, we use (1)-(2), assumptions 1, 4 and the independence of the processes involved to obtain

$$
\begin{aligned}
& E\left[x_{k} y_{k+1}\right]=\pi_{0}^{(k+1)} \bar{\gamma}_{k+1} B_{k} A_{k+1}^{T} H_{k+1}^{T}+\sum_{a=1}^{k \wedge 2} \pi_{a}^{(k+1)} \bar{\gamma}_{k+1-a} A_{k} B_{k+1-a}^{T} H_{k+1-a}^{T}, \\
& E\left[x_{k} y_{k+s}\right]=\sum_{a=0}^{2} \pi_{a}^{(k+s)} \bar{\gamma}_{k+s-a} B_{k} A_{k+s-a}^{T} H_{k+s-a}^{T}, \quad s \geq 2 .
\end{aligned}
$$

Now, from (9), taking into account that $E\left[x_{k} O_{k+s}^{y T}\right]=E\left[\widehat{x}_{k / k+s} O_{k+s}^{y T}\right]$ and denoting $\mathcal{E}_{k, k+s}=E\left[\widehat{x}_{k / k+s} O_{k+s}^{y T}\right]$, we have

$$
E\left[x_{k} \widehat{y}_{k+s / k+s-1}\right]=\mathcal{E}_{k, k+s-1} \mathcal{A}_{k+s}^{T}+\sum_{h=(k+s-3) \vee 1}^{k+s-1} \mathcal{X}_{k, h} \Sigma_{\mu_{h}}^{-1} \mathcal{G}_{k+s, h}, \quad s \geq 1,
$$

and from these two expectations, relation (22) is derived.

Finally, using (20) and (14) for $\widehat{x}_{k / k+s}$ and $O_{k+s}^{y}$, respectively, and taking into account that the innovation is a white process, the recursive relation (23) is obtained. The initial condition is immediately derived from $E\left[x_{k} O_{k}^{y T}\right]=E\left[\widehat{x}_{k / k} O_{k}^{y T}\right]=A_{k} r_{k}^{x y}$.

Remark 2. As commented above, the filtering algorithm obtained in García-Ligero et al. (2018), where the delays are also modelled by a homogeneous Markov chain, cannot be considered for the model at hand due to the influence of gain degradations and because of the noise correlation assumption. The difficulties caused by these factors mainly concern obtaining the one-stage observation predictor in the way specified in expression (9), which provides (12) for the innovation and constitutes the starting point in deriving the proposed algorithms. 


\subsection{Computational procedure}

The computational procedure of the proposed estimators is summarized as follows:

I) Previous matrices (Lemma 3.1.)

From the initial distribution $\pi_{a}^{(1)}, a \in S$, and transition probability matrix $\mathbf{P}$, given in Assumption 4, we obtain $\pi_{a}^{(k)}=P\left(\theta_{k}=a\right), a \in S$ and $\Pi^{(k)}=$ $\operatorname{diag}\left(\pi_{0}^{(k)}, \pi_{1}^{(k)}, \pi_{2}^{(k)}\right) \otimes I_{M}$; these matrices together with $A_{k}, B_{k}, \bar{\gamma}_{k}$ and $R_{k}$, given in assumptions 1-3, determine the matrices $\mathcal{A}_{k}$ and $\mathcal{B}_{k}$ through the expressions established in part $i$ ) of Lemma 3.1, as well as $\mathcal{F}_{k, k-1}, \mathcal{F}_{k, k-2}$ and $\mathcal{F}_{k, k-3}=\mathcal{G}_{k, k-3}$ in part $i i$ ). These elements only depend on the system model information, and so they can be calculated before the observations are available to start the algorithms.

II) Prediction and filtering algorithm (Theorem 4.1.)

At any sampling time $k$, starting with the prior knowledge of the $(k-1)$-th iteration, which provides the scalars $\mathcal{G}_{k-1, k-2}$ and $\mathcal{G}_{k-1, k-3}$, the vectors $J_{k-1}^{x}$ and $J_{k-1}^{y}$, the innovation variance $\Sigma_{\mu_{k-1}}$, the matrices $r_{k-1}^{x}, r_{k-1}^{y}, r_{k-1}^{x y}$, the innovation $\mu_{k-1}$ and the vectors $O_{k-1}^{x}, O_{k-1}^{y}$, the proposed filtering algorithm operates as follows:

a) - Compute $\mathcal{G}_{k, k-1}$ and $\mathcal{G}_{k, k-2}$ by (8).

- Compute $J_{k}^{y}$ by (17). From this, the innovation variance $\Sigma_{\mu_{k}}$ is provided by $(13)$.

- Compute $J_{k}^{x}$ by (16). From this, we obtain $r_{k}^{x}$ by (15); then, the filtering error covariance matrix, $\Sigma_{k / k}$, as well as any prediction error covariance matrix required, $\Sigma_{k+1 / k}, \Sigma_{k+2 / k} \ldots$, are obtained by (11).

- Finally, we obtain $r_{k}^{x y}$ and $r_{k}^{y}$ by (15), which are needed to derive $J_{k+1}^{x}$ and $J_{k+1}^{y}$, respectively, in the next iteration.

b) - When the new measurement $y_{k}$ is available, the innovation $\mu_{k}$ is computed by (12) and, from this, $O_{k}^{x}$ is obtained using (14). Then the filtering estimators $\widehat{x}_{k / k}$, as well as the required prediction estimators, $\widehat{x}_{k+1 / k}, \widehat{x}_{k+2 / k} \ldots$, are computed by (10).

- Finally, in order to obtain $\mu_{k+1}$ in the next iteration, we compute $O_{k}^{y}$ by (14).

III) Fixed-point smoothing algorithm (Theorem 4.2.)

a) Initial conditions: For each fixed sampling point, $k \geq 1$, the initial conditions of the smoothing algorithm are the filter, $\widehat{x}_{k / k}=A_{k} O_{k}^{x}$, the filtering error covariance matrix, $\Sigma_{k / k}=A_{k}\left(B_{k}^{T}-r_{k}^{x} A_{k}^{T}\right)$, and $\mathcal{X}_{k, k}=A_{k} J_{k}^{x}, \mathcal{E}_{k}=A_{k} r_{k}^{x y}$.

b) At the sampling time $k+s$, with $s=1,2, \ldots$, run the filtering algorithm until time $k+s$; then the proposed smoothing algorithm operates as follows:

- For each fixed $k \geq 1$ and $s=1,2, \ldots$, compute $\mathcal{X}_{k, k+s}$ by (22). From this, the smoother, $\widehat{x}_{k / k+s}$, and its error covariance matrix, $\Sigma_{k / k+s}$, are obtained by (20) and (21), respectively.

- For the next step, $\mathcal{E}_{k, k+s}$ is obtained by (23).

\section{Simulation results}

In this section, a numerical simulation example shows the effectiveness of the prediction, filtering and fixed-point smoothing proposed algorithms. To do so, a scalar signal 
is considered and the prediction, filtering and fixed-point smoothing error variances are used to measure the estimators' accuracy. The quality of the estimators is analysed versus different probability distributions for the random variables modelling the sensor gain degradation, different values of measurement noise correlation and different transition probability matrices for the Markov chain modelling the delays.

As in García-Ligero et al. (2018) let us consider a zero-mean scalar signal $\left\{x_{k}, k \geq 1\right\}$ with covariance function

$$
E\left[x_{k} x_{h}\right]=1.025641 \times 0.95^{k-h}, \quad 1 \leq h \leq k,
$$

which is clearly factorised, according to Assumption 1, as

$$
A_{k}=1.025641 \times 0.95^{k} \text { and } B_{h}=0.95^{-h} .
$$

The measurement outputs are affected, as in (1), by multiplicative and additive processes. We start by assuming that the process $\left\{\gamma_{k}, k \geq 1\right\}$ is a white sequence of scalar random variables with $P\left(\gamma_{k}=0\right)=0.1, P\left(\gamma_{k}=0.5\right)=0.4$ and $P\left(\gamma_{k}=1\right)=0.5$. The measurement noise is defined as $v_{k}=c\left(\nu_{k}+\nu_{k+1}\right)$, where $\left\{\nu_{k}, k \geq 1\right\}$ is a zeromean Gaussian white process with variance $E\left[\nu_{k}^{2}\right]=0.5, k \geq 1$. Then, $E\left[v_{k}^{2}\right]=c^{2}$, $E\left[v_{k} v_{k-1}\right]=0.5 c^{2}$ and, consequently, the parameter $c$ determines the autocovariance function of the measurement noise $\left\{v_{k}, k \geq 1\right\}$.

Also, we assume that the available signal measurements can be delayed by one or two sample periods during the transmission; that is, the processed observations are modelled by (2), where, as established in Assumption 4, the delay is modelled by a homogeneous Markov chain, $\left\{\theta_{k}, k \geq 1\right\}$, with initial distribution $\pi_{0}^{(1)}=1$, and $\pi_{1}^{(1)}=\pi_{2}^{(1)}=0$ (the first observation is not delayed) and transition probability matrix

$$
\mathbf{P}=\left(\begin{array}{ccc}
0.99 & 0.003 & 0.007 \\
0.01 & 0.98 & 0.01 \\
0.11 & 0.02 & 0.87
\end{array}\right)
$$

Figure 1 shows the prediction, filtering and fixed-point smoothing error variances, $\Sigma_{k / k+s}, s=-3,-1,0,1,3,6,9$, when $c=0.75$. This figure reveals that the performance of the estimators improves as more observations are used; that is, the error variances of the smoothers are less than the filtering error variances, which, in turn, are less than those of the predictors. Hence, the accuracy of the smoothers is higher than that of the filter and predictors. Furthermore, it is noted that the values of the fixed-point smoothing error variance decrease with increasing $s$, although this decrease becomes almost negligible for $s>9$.

Next, again assuming $c=0.75$ and the transition matrix $\mathbf{P}$, the influence of the sensor gain degradation phenomenon is analysed considering different distributions of probability for the random variables representing this degradation. Specifically, we again consider a white sequence of random variables, $\left\{\gamma_{k}, k \geq 1\right\}$, with values $0,0.5$, 1 , and $P\left(\gamma_{k}=0\right)=0.1$. In this situation, the increase of $P\left(\gamma_{k}=1\right)$ means that there is less degradation and, consequently, more information on the signal is received at the sensor. Figure 2 shows the filtering error variances for the values $P\left(\gamma_{k}=1\right)=0.1,0.3$, $0.5,0.7,0.9$; it can be seen that the filtering error variances decrease when $P\left(\gamma_{k}=\right.$ 1) increases. Hence, in accordance with the intuitive view, the filtering performance 


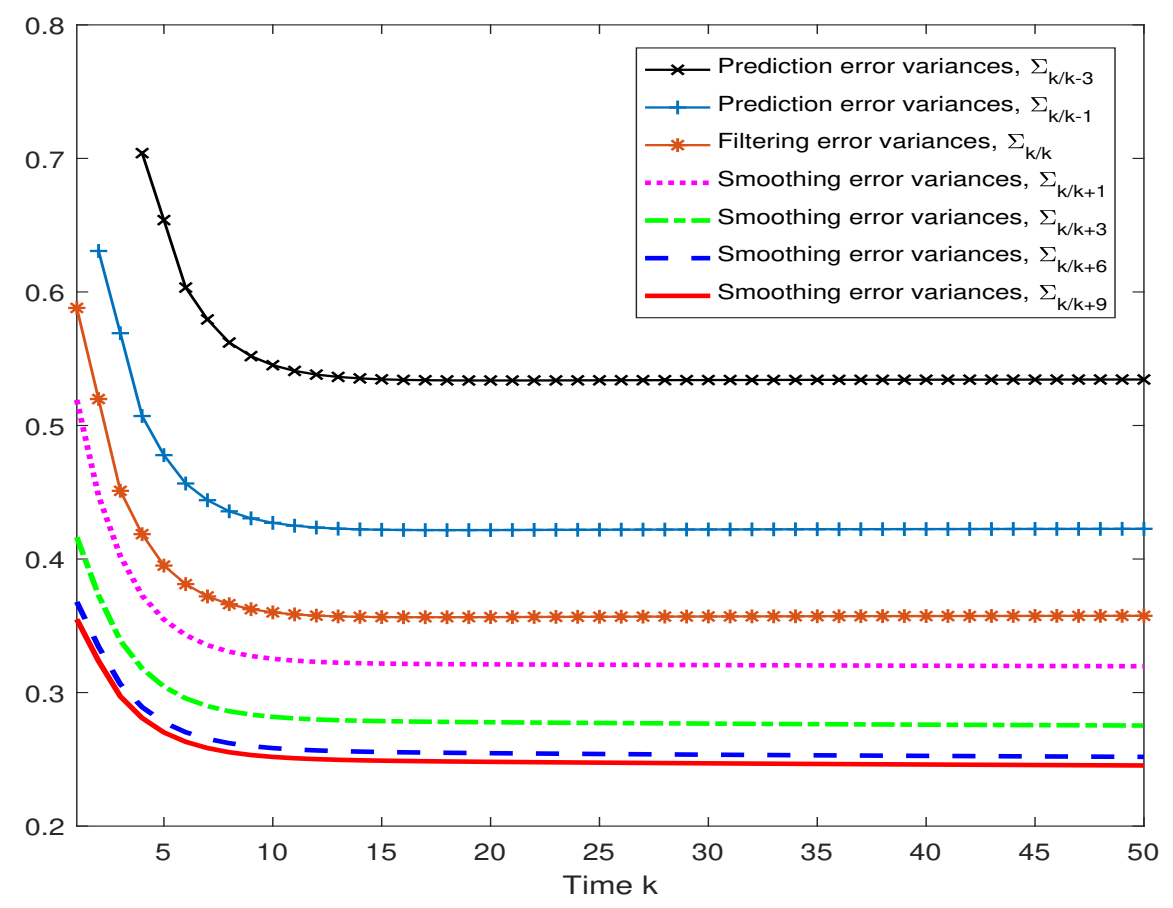

Figure 1. Prediction, Filtering and fixed-point smoothing error variances when $c=0.75$

improves when there is less signal degradation.

In order to study the influence of the correlation of measurement noise, we analyse the filtering error variances for different values of $c$, thus obtaining different autocovariance functions of measurement noise. Specifically, Figure 3 shows the performance of the proposed filter for the values $c=0.25,0.5,0.75$ and 1 . This figure reveals that when $c$ decreases, so do the corresponding filtering error variances and, therefore, the performance of the estimators improves.

We also analysed the performance of the proposed estimators when the delay is modelled by different Markov chains. To do so, the filtering error variances were calculated assuming $c=0.75$, the same initial distribution (in the first observation there is no delay) and the following transition probability matrices:

$$
\mathbf{P}_{1}=\left(\begin{array}{ccc}
0.95 & 0.03 & 0.02 \\
0.05 & 0.89 & 0.06 \\
0.07 & 0.01 & 0.92
\end{array}\right), \quad \mathbf{P}_{\mathbf{2}}=\left(\begin{array}{ccc}
0.89 & 0.07 & 0.04 \\
0.055 & 0.89 & 0.055 \\
0.07 & 0.05 & 0.88
\end{array}\right) \text {. }
$$

The properties of the Markov chains lead us to conclude that the no delay probabilities converge to constant values; in our case these values are $0.79,0.55$, and 0.35 , for the transition probability matrices considered, $\mathbf{P}, \mathbf{P}_{\mathbf{1}}$ and $\mathbf{P}_{\mathbf{2}}$, respectively. Therefore, as expected, Figure 4, which shows the filtering error variances for these models, reveals that as the limit probability of no delay increases, the filtering error variances become smaller and, consequently, the performance of the estimators improves. 


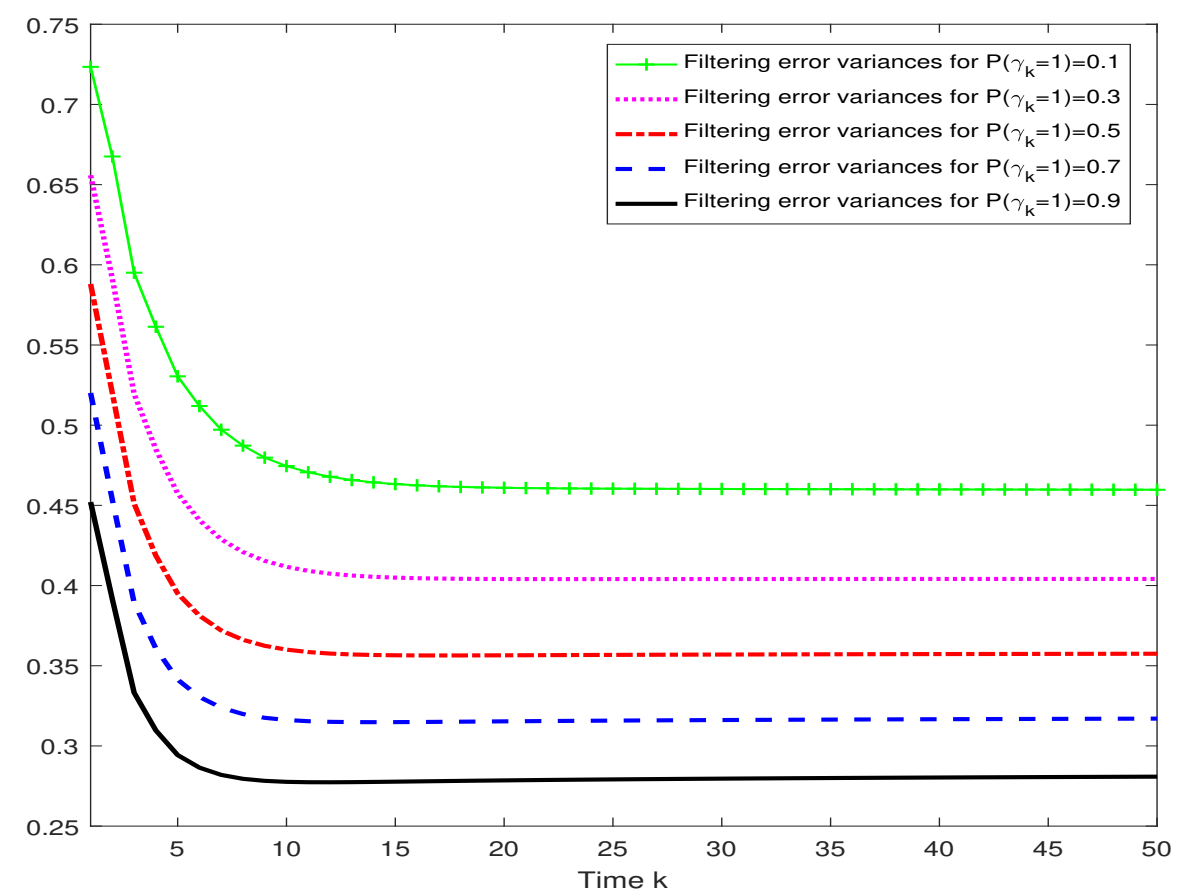

Figure 2. Filtering error variances for different values of $P\left(\gamma_{k}=1\right)$

\section{Conclusions}

In this paper, we investigate the linear estimation of a signal from measurements with stochastic sensor gain degradation and correlated noises, subject to random delays during transmission. The sensor gain degradation is modelled by a white sequence random variables with values in $[0,1]$, which is an extension of the missing measurement model. The measurement noise is correlated at consecutive sampling times, which is a valid assumption in many practical situations. The processed measurements can suffer delays. In our approach, unlike that adopted in previous papers, these are not modelled by independent random variables, but by a homogeneous Markov chain which model a possible correlation of delays at different sampling times. Therefore, we propose a general model which is of greater applicability to real situations. In this context, the derivation of the prediction, filtering and fixed-point smoothing algorithms is only based on the information provided by the first and second-order moments of the processes involved in the model. The proposed estimation algorithms have the advantage that they are applicable when the state-space model is known.

An immediate area of interest for future research is to use the current observation model with sensor gain degradation and transmission delays modelled by Markov chains in multisensor network systems, addressing fusion estimation problems and comparing the performance of different fusion estimators. Another challenging topic would be to consider bounded delays of more than two sample periods, described by Markov chains whose state space, finite, reflects the maximum delay allowed. Finally, an interesting extension of the current research could be to address the joint signal and fault estimation for the systems considered in this paper, which has recently been 


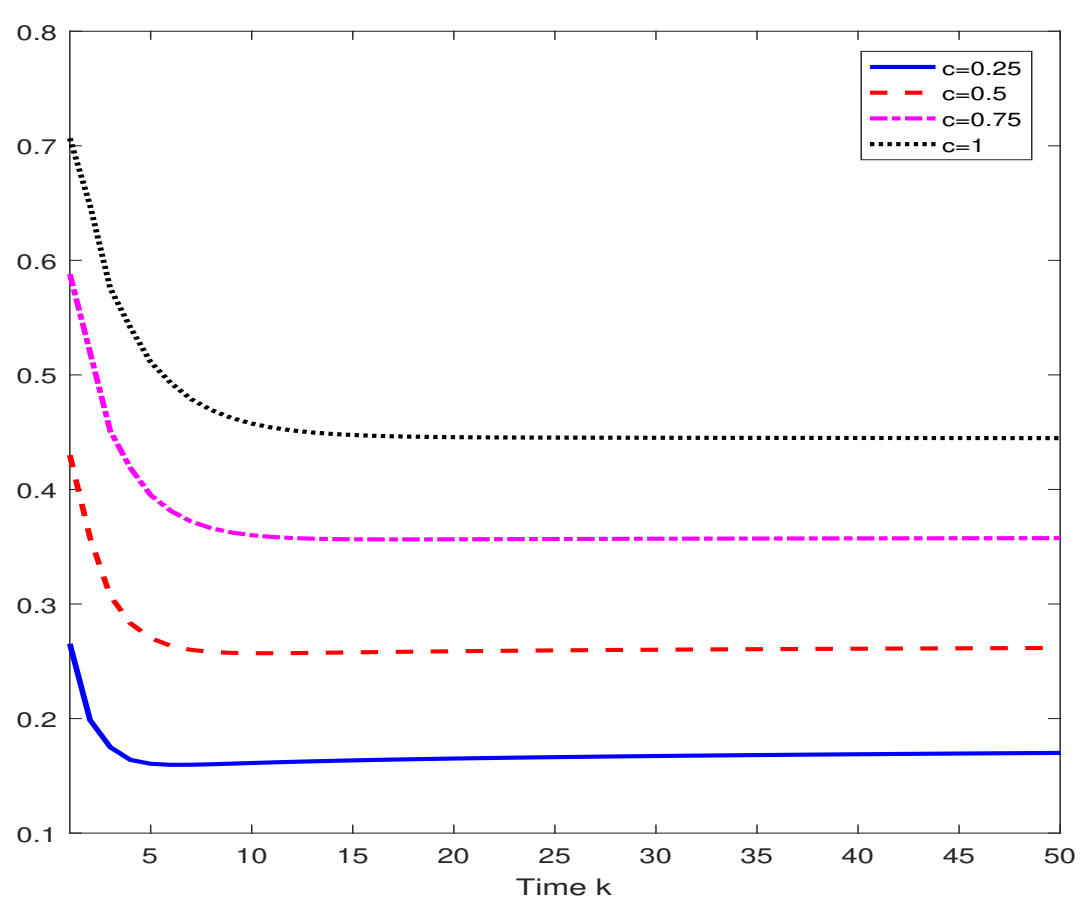

Figure 3. Filtering error variances when $c=0.25,0.5,0.75,1$

investigated by $\mathrm{Hu}$, Wang \& Gao (2018) for a class of uncertain nonlinear systems with random faults and sensor saturations.

\section{Disclosure statement}

No potential conflict of interest was reported by the authors.

\section{Funding}

This research is supported by Ministerio de Economia, Industria y Competitividad, Agencia Estatal de Investigación and Fondo Europeo de Desarrollo Regional FEDER (grant no. MTM2017-84199-P).

\section{Notes on contributors}

M. J. García-Ligero received her M.Sc. degree in Mathematics in 1985 and her Ph.D. in Linear estimation in stochastic systems with distributed parameters by uncertain observations in 1995, both from the University of Granada (Spain). She is currently a Associate professor at the Department of Statistics and Operations Research, University of Granada (Spain). Her main research interests are stochastic systems and signal estimation. 


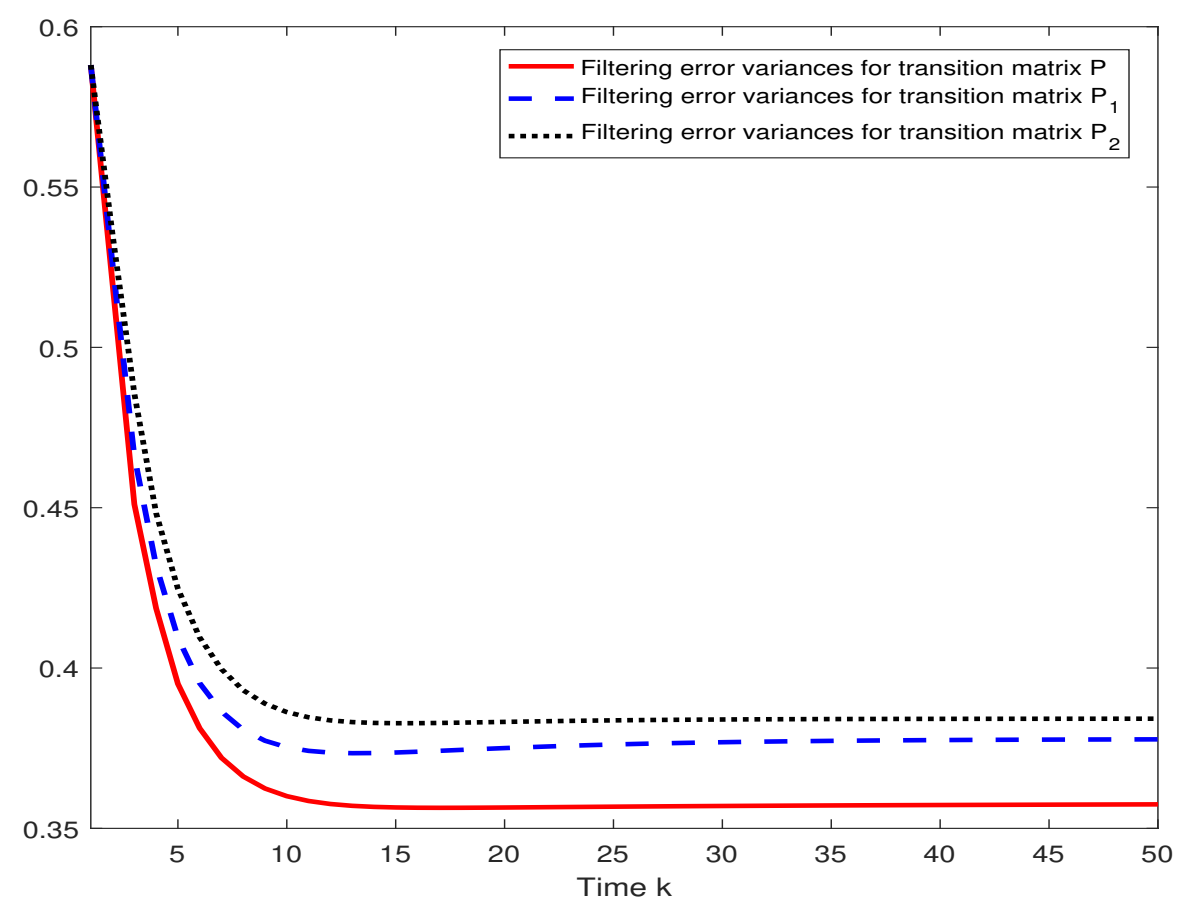

Figure 4. Filtering error variances for different transition probability matrices

A. Hermoso-Carazo received her MSc degree in Mathematics (Statistics) and her PhD in Likelihood Test in Log Normal Processes, both from the University of Granada (Spain) in 1979 and 1984, respectively. She is currently a professor at the Department of Statistics and Operations Research, University of Granada (Spain). Her current research interests include stochastic systems, filtering, prediction and smoothing.

J. Linares-Pérez received her MSc degree in Mathematics (Statistics) and her PhD in Stochastic Differential Equations, both from the University of Granada (Spain) in 1980 and 1982, respectively. She is currently a professor at the Department of Statistics and Operations Research, University of Granada (Spain). Her research interest involves the areas of stochastic calculus and estimation in stochastic systems.

\section{Orcid}

M. J. García-Ligero, http://orcid.org/0000-0001-5604-5599

A. Hermoso-Carazo, http://orcid.org/0000-0001-8120-2162

J. Linares-Pérez, http://orcid.org/0000-0002-6853-555X

\section{References}

Caballero-Águila, R., Hermoso-Carazo, A., Jiménez-López, J.D., Linares-Pérez, J. \& Nakamori, S. (2010). Signal estimation with multiple delayed sensors using covariance information. Digital Signal Processing, 20, 528-540. 
Caballero-Águila, R., Hermoso-Carazo, A., Linares-Pérez, J. \& Nakamori, S. (2013). Linear estimation based covariances for networked systems featuring sensor correlated random delays. International Journal of Systems Science, 44(7), 1233-1244.

Caballero-Águila, R., Hermoso-Carazo, A. \& Linares-Pérez, J. (2017a). Fusion estimation from multisensor observations with multiplicative noises and correlated random delays in transmission. Mathematics, 2017, 5, 45.

Caballero-Águila, R., Hermoso-Carazo, A. \& Linares-Pérez, J. (2017b). Optimal fusion estimation with multi-step random delays and losses in transmission. Sensors, 2017, 17, 1151.

Caballero-Águila, R., Hermoso-Carazo, A. \& Linares-Pérez, J. (2017c). Least-Squares filtering algorithm in sensor networks with noise correlation and multiple random failures in transmission. Mathematical Problems in Engineering, 201\%, Article ID 1570719.

Caballero-Águila, R., Hermoso-Carazo, A., Linares-Pérez, J. \& Wang, Z. (2019). A new approach to distributed fusion filtering for networked systems with random parameter matrices and correlated noises. Information Fusion, 45, 324-332.

Feng, J., Wang, Z. \& Zeng, M. (2013). Distributed weighted robust Kalman filter fusion for uncertain systems with autocorrelated and cross-correlated noises. Information Fusion, 14, $76-86$.

Gao, S. \& Chen, P. (2014). Suboptimal filtering of networked discrete-time systems with random observation losses. Mathematical Problems in Engineering, 2014, Article ID 151836.

García-Ligero, M. J., Hermoso-Carazo, A. \& Linares-Pérez, J. (2011). Estimation for discretetime systems with multiple packet dropouts using covariance information. Mathematical and Computer Modelling, 54, 2277-2286.

García-Ligero, M. J., Hermoso-Carazo, A. \& Linares-Pérez, J. (2015). Distributed fusion estimation in networked systems with uncertain observations and Markovian random delays. Signal Processing, 106, 114-122.

García-Ligero, M. J., Hermoso-Carazo, A. \& Linares-Pérez, J. (2017). Estimation from a multisensor environment for systems with multiple packet dropouts and correlated measurement noises. Applied Mathematical Modelling, 45, 324-332.

García-Ligero, M. J., Hermoso-Carazo, A. \& Linares-Pérez, J. (2018). Covariance-based leastsquares filtering algorithm under Markovian measurement delays. International Journal of Computer Mathematics, https://doi.org/10.1080/00207160.2017.1422496.

Guo, Y. (2017). Switched filtering for networked systems with multiple packet dropouts. Journal of the Franklin Institute, 354, 3134-3151.

Han, C. \& Zhang, H. (2009). Linear optimal filtering for discrete-time systems with random jump delays. Signal Processing, 89, 3097-3104.

Han, C., Zhang, H. \& Fu, M. (2013). Optimal filtering for networked systems with Markovian communication delays. Automatica, 49, 3097-3104.

He, X., Wang, Z. \& Zhou, D. (2009). Robust $H_{\infty}$ filtering for time-delay systems with probabilistic sensor faults. IEEE Signal Processing Letters, 16, 442-445.

Hounkpevi, F. O. \& Yaz, E. E. (2007). Minimum variance generalized state estimators for multiple sensors with different delay rates. Signal Processing, 87, 602-613.

Hu, J., Wang, Z., Gao, H. \& Stergioulas, L. K. (2012). Extended Kalman filtering with stochastic nonlinearities and multiple missing measurements. Automatica, 48, 2007-2015.

Hu, J., Wang, Z. \& Gao, H. (2013). Recursive filtering with random parameter matrices, multiple fading measurements and correlated noises. Automatica, 49, 3440-3448.

Hu, J., Wang, Z. \& Gao, H. (2018). Join state and fault estimation for time-varying nonlinear systems with randomly occurring faults and sensor saturations. Automatica, 9\%, 150-160.

Li, N., Sun, S. \& Ma, J. (2014). Multi-sensor distributed fusion filtering for networked systems with different delay and loss rates. Digital Signal Processing, 34, 29-38.

Liu, Y., Wang, Z. \& Zhou, D. (2014). Optimal filtering for networked systems with stochastic sensor gain degradation. Automatica, 50, 1521-1525.

Liu, Y., Wang, Z., He, X. \& Zhou, D. H. (2016). Minimum-variance recursive filtering over sensor with stochastic sensor gain degradation: Algorithms and performance analysis. IEEE Transaction Control Network Systems, 3(3), 265-274. 
Pang, C. \& Sun, S. (2015). Fusion predictors for multisensor stochastic uncertain systems with missing measurements and unknown measurement disturbance. IEEE Sensors Journal, $15(8), 4346-4354$.

Song, H., Yu, L. \& Zhang W. A. (2009). $H_{\infty}$ filtering of network-based systems with random delay. Signal Processing, 89, 615-622.

Sun, S., Xie, L., Xiao, W. \& Soh, Y. C. (2008). Optimal linear estimation for systems with multiple packet dropouts. Automatica, 44(5), 1333-1342.

Sun, S. \& Tian, T. (2011). Optimal linear estimators for systems with random measurement delays. Journal of Control Theory and Applications, 9, 76-82.

Sun, S. \& Xiao, W. (2013). Optimal linear estimators for systems with multiple random measurement delays and packet dropouts. International Journal of Systems Science, 44(2), $358-370$.

Sun, S., Tian, T. \& Lin, H. (2017). State estimators for systems with random parameter matrices, stochastic nonlinearities, fading measurements and correlated noises. Information Sciences, 397-398, 118-136.

Tian, T., Sun, S. \& Li, N. (2016). Multi-sensor information fusion estimators for stochastic uncertain systems with correlated noises. Information Fusion, 27, 126-137.

Tian, T., Sun, S. \& Lin, H. (2019). Distributed fusion filter for multi-sensor systems with finite-step correlated noises. Information Fusion, 46, 128-140. 